\title{
El final del reino Antiguo egipcio. Cambios y constantes
}

\author{
Antonio Pérez Largacha *
}

\section{RESUMEN}

El final del Reino Antiguo ha sido explicado desde perspectivas económicas - un agotamiento de los recursos ante el crecimiento de las instituciones funerarias- 0 consecuencia del aumento de poder de los nomarcas que llevó a una descentralización del país. Sin embargo, lo que implica el término crisis no ha sido analizado desde una perspectiva egipcia, al mismo tiempo

que no se ha tenido en cuenta el propio dinamismo de la cultura egipcia, los cambios que se producen en su entorno -próximo y lejano-o o las características internas de una cultura que, lejos de ser monolítica a lo largo de su desarrollo, experimenta importantes transformaciones.

\section{ABSTRACT}

The end of the Old Kingdom has been explained from a economic perspective - the exhaustion of the resources as consecuence of the growth of the funeral institutions - or consequence of the increase of power of the nomarcas that culminated in the decentralization of Egypt. However, what implies the term crisis has not been analyzed from a Egyptian perspective, at the same time that have not been kept in mind the own dynamism of the Egyptian culture, the changes that takes place in its environment, internal or external, or the characteristics of a culture that, far from being monolithic along its development, it experiences important transformations.

* Universidad Alcalá de Henares. 
A diferencia del mundo romano, y posiblemente del maya, los períodos de crisis, colapso, declive o desaparición que han experimentado diferentes civilizaciones, como la egipcia tras la llamada época de las pirámides, no han suscitado la atención de la investigación. En el caso del mundo faraónico, las razones del diferente interés que, por ejemplo, han tenido sus períodos de descomposición con aquellos otros de formación - como el propio surgimiento del Estado, la creación de un Imperio tras el llamado período Hikso o la dinastía Saíta, llamada también el último resurgimiento egipcio-, son variadas, influyendo tanto el volumen y carácter de la información disponible como, principalmente, la idea preconcebida de que el mundo egipcio siempre fue capaz de recuperarse y de generar nuevos períodos históricos que, como el Reino Medio, es considerado el clasicismo egipcio y no muy alejado de lo que había sido característico con anterioridad. Es decir, en el fondo de estos planteamientos subyace la idea de que lo egipcio permaneció, no cambió sustancialmente, considerándose el llamado Primer Período Intermedio como una transición, inevitable en cierta medida si aceptamos el carácter cíclico que ya los griegos tenían de las culturas, pero que no supuso cambios fundamentales, ya que si bien las pirámides dejaron de ser los monumentos funerarios por excelencia, la momificación, la religión, el carácter divino del rey, el arte... siguieron estando latentes en una cultura que no es capaz, fuera de la egiptología contemporánea, de mostrar la variedad, la evolución y los cambios que se producen entre un período y otro de su dilatada historia e, incluso, entre una dinastía y otra ${ }^{1}$.

Esta diferente actitud entre los estudios tendentes a establecer las bases de una civilización, o de un Estado, y aquellos dedicados a los períodos de crisis, son variadas, influyendo que siempre es más gratificante analizar aquello que germina en algo palpable, en el caso del mundo faraónico hasta colosal y exótico, y no aquellos períodos de declive y crisis en los que esos logros, hitos, acontecimientos y personajes que marcan un período, y hasta una cultura, llegan a su declive o final. Una excepción en este tipo de estudios es la del Imperio Romano y, especialmente, los cambios que acontecieron en su mitad occidental y que germinaron en Estados, reinos y pueblos sobre los que se construyó la Europa medieval de la que somos herederos pero, incluso en un período en el que la información es muy abundante y que marca en cierta medida nuestra con-

A ello ha contribuido el que, a pesar de que desde sus comienzos la egiptología estuvo ligada a la arqueología bíblica como a la naciente Asiriologia, con el paso del tiempo estas disciplinas fueron labrándose caminos diferentes que solo en los últimos años comienzan a acercarse (Pérez Largacha, 1998). 
ciencia occidental, la explicación de los invasiones bárbaras ha sido la dominante durante siglos, posiblemente por ser difícil de asumir que el declive interno de una cultura emblemática pudiera también coadyuvar a su colapso.

Por tanto, todos aquellos períodos en los que se produce un estancamiento, sea este definitivo o una transición hacia un revitalizamiento, son poco atractivos y en ellos el investigador, muchas veces de forma inconsciente, vuelca todo lo que conllevan adjetivos y calificaciones como crisis, declive, colapso o desaparición, adoptando en muchas ocasiones explicaciones simplistas y generales, por lo general invasiones de pueblos bárbaros y desastres naturales, para explicar dichos procesos, sin pararse a considerar la riqueza interna de estos períodos. Ello se debe, en gran medida, al hecho de que es difícil para el investigador, y la sociedad para la que escribe, plantear cómo algo que ha llegado a florecer puede desaparecer o decaer, siendo más fácil buscar las explicaciones en factores externos, ajenos en muchas ocasiones a la dinámica cultural interna. Así, en el caso de Egipto la realeza volvió a aparecer, la religión, los templos y el ejército fueron reapareciendo con mayor peso especifico, por lo que la crisis fue aprovechada, y estuvo caracterizada, por la penetración de poblaciones nómadas, bárbaras. Pero en pocas ocasiones se plantea si ese posterior florecimiento fue una consecuencia de los cambios, los impulsos y las adaptaciones acontecidas durante esa transición, asumiéndose en la mayoría de las ocasiones que implican una vuelta a un pasado (Assman, 1995). La crisis del siglo Ill del Imperio Romano y la progresiva aparición de pueblos bárbaros puede considerarse como el paradigma histórico de esta problemática, olvidándose la capacidad de autogeneración, de cambio y de evolución interna que la propia sociedad romana desarrolló, no solo con las reformas de Diocleciano, sino también con posterioridad, así como las propias aportaciones que realizaron esos pueblos gérmanos que, al igual que los odiados asiáticos de Egipto, estuvieron mucho tiempo en contacto con la «civilización» antes de ser considerados causantes de su muerte y agonía ?

Es por ello que en las siguientes páginas nuestra intención será mostrar las causas que llevaron a la crisis del Reino Antiguo, las cuales están ya muy lejos de la interpretación tradicional de una pérdida de poder de la

2 Durante décadas ha sido normal considerar el que los egipcios podían, y tenían, relaciones con el exterior, construir edificios, mantener guarniciones y vivir en el extranjero, pero lo contrario era una premisa ni siquiera planteada, siendo lo que los últimos hallazgos arqueológicos en el Delta están poniendo de relieve, no solo en los períodos intermedios, también en los de teorica expansión y centralización. 
realeza ante una aristocracia, cortesana y provincial, cada vez más independiente. Comprensión del problema que se nos revela como fundamental para conocer la dinámica posterior del mundo faraónico que, por ejemplo, ya no desarrollará la imagen de un rey divino (Posener, 1960; Barta, 1975; O'Connor \& Silverman, 1995), de una sociedad que no alcanza los niveles de centralización desarrollados durante las dinastías III-V, de un mundo más abierto y receptivo y cuya historia y evolución están cada vez más interrrelacionadas con la de sus vecinos próximo orientales, no solo la región Sirio-palestina, sino también del mundo que los griegos denominaron genéricamente como Mesopotamia (Pérez Largacha, en prensa).

Por otra parte, y ésta es una crítica que puede extenderse a toda la egiptología desarrollada hasta la década de los 80 , la reconstrucción histórica se venía realizando desde una literalidad de los textos, sin proceder a un análisis interno y externo de las fuentes; cuándo, por qué y para qué fueron redactados unos textos que, por otra parte, solamente podían ser leídos por el $1 \%$ de la población (Baines \& Eyre, 1987) ${ }^{3}$. Así, la conocida descripción realizada por Ipuwer, teóricamente de la situación interna de Egipto durante el Primer Período Intermedio, ha sido tomada como reflejo literal de las consecuencias que para un Estado como el Faraónico suponía la ausencia de una monarquía y de una administración centralizada que mantuviera una política hidráulica sobre la que descansaba la prosperidad de Egipto, sin olvidar las connotaciones de "revolución social» que la historiografía marxista quiso ver en lo relatado. Sin embargo, son muy pocos los que han señalado sus exageraciones e incorrecciones históricas, lo que permite entender el texto como un ejercicio literario en el que el autor lleva hasta el límite las consecuencias de una mala administración y de una situación económica verdaderamente preocupante, pero todo ello con vistas a reclamar una vuelta de la realeza capaz de terminar con el caos y reestablecer el orden, no pudiendo olvidar que la realeza emergente surgió de una guerra civil y, en segundo lugar, de una ciudad que hasta entonces no había tenido importancia, Tebas, debiendo proceder a una legitimación de la misma, pero siempre teniendo presente que la culpa nunca había sido de los reyes que gobernaron el país (Griffiths, 1991), y sí del abandono de una institución, la realeza, que al contrario que en Mesopotamia no «descendió» del cielo, sino que estuvo presente ya en la creación del orden cósmico en contraposición a isfet, el desorden.

3 Como suele acontecer ante la revisión historiográfica de todo período, en el caso de la egiptología se ha pasado de una excesiva dependencia en las fuentes, creyendo todo aquello que estas reflejaban, a otra tendencia en la que ningún texto es considerado si no esta confirmada su información mediante otro texto externo o un hallazgo arqueológico. 
Otro de los problemas es el de ubicar fechas o acontecimientos concretos que delimiten, en su inicio o final, estos períodos de crisis. Un ejemplo claro es el del Imperio Romano donde se ha establecido otro período conocido como Antigüedad Tardía. En el caso concreto que nos ocupa, el declive del Reino Antiguo suele ubicarse en la VI-VII dinastías, aunque también se ha apuntado que es a partir de la $V$ dinastía cuando comienzan a observarse cambios que irán poniendo las bases de la posterior quiebra de una sociedad y una administración que había sido capaz de construir las pirámides. Sin embargo, hablar de crisis a partir de la $V y$, especialmente, desde la VI es, en nuestra opinión, muy difícil si tenemos en cuenta que: A) la VI dinastía dura cerca de 200 años, en líneas generales el mismo período de tiempo que transcurre entre la crisis del siglo III y, pongamos por caso, el 406 con la invasión de los pueblos bárbaros, siendo muchos los factores de cambio, revitalizamiento que acontecieron en el mundo romano en dicho lapso de tiempo, B) La VI dinastía constituye nuestra principal fuente de información, no solo a nivel arqueológico, funerario, sino también desde el punto de vista del funcionamiento de las provincias, constatándose además un desarrollo de la escritura y de las relaciones internacionales, en especial con Ebla y Biblos, ¿es ello reflejo de un tiempo de crisis?

La intención de las próximas páginas es enmarcar, a veces desde un punto de vista antropológico, las razones que llevaron al colapso del Reino Antiguo y analizar si en verdad puede hablarse de crisis, por lo que también deberemos hacer referencia a qué entendemos por esos conceptos. El planteamiento que se esboza, y que tendrá su continuación en próximos trabajos, se enfrenta a uno de los mayores retos de la historiografía; siempre es más fácil desmontar una hipótesis que construir un nuevo marco conceptual, intentando este trabajo esbozar algunas líneas de la nueva reconstrucción histórica de un período que cada vez resulta más ilustrativo y que, paradójicamente, ha sido más estudiado desde la arqueología bíblica que desde la egiptología ${ }^{4}$.

Una de las tendencias historiográficas, y que ha pasado al conjunto de la sociedad, ha sido la de juzgar la importancia de una cultura por los restos arqueológicos que nos ha legado y su monumentalidad, siendo por

\footnotetext{
4 No hemos de olvidar la polemica suscitada sobre la ubicación histórica de los movimientos patriarcales, que algunos ponen en relación con los movimientos de pueblos Amorreos que acontecen en este periodo.
} 
ello que la imagen para el período que nos ocupa resulta desalentadora: las pirámides fueron disminuyendo en tamaño, calidad y número a partir de la $V$ dinastía (Lehner, 1997), al mismo tiempo que del llamado Primer Período Intermedio apenas conservamos restos monumentales, lo que origina una sensación de declive cultural. Sin embargo, a excepción de las pirámides, las necrópolis reales y de los templos de Tebas, nuestro conocimiento sobre la cultura material del Egipto faraónico es prácticamente nulo y, cuando algún hallazgo es realizado fuera de los tradicionales centros que han centrado la investigación arqueológica, las sorpresas pueden ser mayúsculas ${ }^{5}$. En lo que respecta al primer período intermedio, la capital de Egipto por entonces, Heracleopolis, es prácticamente desconocida ${ }^{6}$ pero, como ya hemos señalado, desde la $V y$, especialmente, la VI dinastía, nuestras fuentes de información son mucho más variadas y ricas, ¿se corresponde ello a un período de crisis? Así, el número de tumbas conocidas es mayor $y$, aunque en las mismas puede detectarse cierto declive artístico respecto a las grandes tumbas nobiliarias de la V y VI dinastías, también es cierto que se producen avances, como los primeros Textos de los Sarcófagos (Hoffmeier, 1991), se producen diferencias regionales (Bourriau, 1991), artísticamente encontramos una mayor variedad de temas y mayor libertad del artista...

Siguiendo lo esbozado, se ha tendido a considerar la IV dinastía como una de las más gloriosas de la civilización egipcia, pero de ella apenas conocemos los nombres de algunos reyes y, por supuesto, sus complejos piramidales, siendo además una sociedad encerrada en la corte menfita, sin apenas relaciones con el exterior (Pérez Largacha, 1998b). Es cierto que la centralización de recursos, económicos y humanos, fue la más importante pero, ¿hemos de medir la grandeza de un período por el volumen y tamaño de sus construcciones?, ¿por su grado de centralización?, ¿no es mucho más rica y variada la cultura que se enfrenta a problemas más allá de su corte o de las preocupaciones funerarias de sus reyes? ${ }^{7}$.

5 El caso de Avaris es el mejor conocido (Bietak, 1996), siendo, por otra parte, ejemplo de como puede ser modificada la visión, riqueza e importancia de un periodo de la historia de Egipto considerado, por los propios egipcios, como de declive. Pero tampoco podemos olvidar los ejemplos de Elefantina, de Buto o de Qantir, por mencionar aquellos en los que la investigación lleva trabajando un mayor número de años.

6 Los trabajos de excavación se iniciaron con Petrie y en los últimos años las investigaciones de la misión española se han centrado en la necrópolis del Tercer Período Intermedio y, cuando se han realizado prospecciones o pequeñas campañas en el área del periodo que nos ocupa, la belleza, calidad y riqueza documental sorprende.

7 La reciente exposición celebrada en París sobre el Egipto de las Pirámides resulta ilustrativa en el sentido de que la mayoría de las piezas proceden de la $\vee$ y VI dinastías. 
Es por ello importante delimitar que entendemos por colapso o crisis, ya que los períodos así calificados suelen ser más variados en todas sus manifestaciones encontrando, por ejemplo, el artista una mayor libertad para expresar bien sus ideas o las que debe expresar siguiendo los deseos de los gobernantes, aspecto determinante no solo en el mundo egipcio (Gaballa, 1976). Incluso en el campo religioso encontramos una mayor diversidad, ya no solo con la llamada democratización del culto funerario, también en la magia, en la religiosidad popular que, aunque difícil de rastrear, comienza a mostrarnos sus primeras manifestaciones (Baines, 1987).

Han sido pocos los que se han preocupado por el problema del colapso de las civilizaciones, siendo uno de ellos Toymbie con su conocida formulación de cambio-respuesta. La crisis, su duración, intensidad y carácter depende del modo en el que la sociedad de respuesta a unas nuevas condiciones que se producen en su entomo, sin establecer si priman los factores externos o internos. Es decir, en el caso de la civilización egipcia tendríamos un período de centralización y de esplendor que encuentra en las pirámides su principal exponente para, posteriormente, con el Reino Medio experimentar un nuevo renacer sobre las cenizas pero, ¿dicha respuesta fue a partir del pasado o de los cambios y adaptaciones que vivió Egipto desde la $V$ dinastía? Como veremos en las siguientes páginas, fueron tanto factores externos como internos los que propiciaron los cambios que se producen desde la $V$ dinastía pero, ¿deben considerarse dichos cambios reflejo de una crisis? o, por el contrario, ¿dichos cambios son reflejo de un enriquecimiento, de unos nuevos valores que permiten el desarrollo de una nueva fase cultural conocida como el clasicismo egipcio?

Antes de continuar debemos detenernos en otro aspecto importante y ya enunciado. Cuando hablamos de la historia de Egipto la dividimos en periodos que, por lo general, suelen considerarse unitarios; el Reino Antiguo con las pirámides, el Reino Medio con su arte y literatura, el Reino Nuevo con la creación de un Imperio y unos reyes guerreros y expansionistas que llevan a Egipto al cenit de su evolución, ¿podemos seguir trabajando con estos conceptos?, ¿se admitiría entre los investigadores del mundo helenístico estudiarlo desde la globalidad? o, ¿es lo mismo la llamada dinastía julio-claudia que la de los Antoninos? Lógicamente no. Existen circunstancias y cambios importantes a lo largo de períodos tan amplios de la historia, del mismo modo que la III dinastía en nada se parece a la $V y$, mucho menos, a la $V I$, pero todas ellas se engloban como Reino Antiguo. Muchas veces trabajamos con esquemas mentales y temporales heredados de una historiografía, ni siquiera de las propias civilizaciones que son objeto de estudio (Redford, 1986), olvidando los riesgos que ello entraña, ¿son lo mismo los primeros y los segundos palacios 
minoicos?; tienen el mismo substrato cultural y étnico, pero sus manifestaciones y actitudes son diferentes en muchos y variados aspectos, por lo que no podemos hablar de una cultura minoica en sentido global, como tampoco de un Reino Antiguo con unas características similares a lo largo de todo su desarrollo temporal.

Uno de los factores que se pone en relación con el final de muchas fases culturales de la humanidad son los llamados desastres naturales ${ }^{8}$. Esta línea de investigación, que se encuadra en el modelo evolucionista entre otros de Service (1981), es acorde con la visión ya mencionada de los griegos, y de nuestra sociedad, de un carácter cíclico de las civilizaciones ${ }^{9}$. En el caso de Egipto, los estudios de Bell (1971), pusieron las bases para poder hablar de un descenso generalizado en el nivel de las crecidas del Nilo (Hassan, 1997), lo que, lógicamente, perturbaría a una sociedad y una economía como la egipcia basada en una agricultura dependiente de las crecidas anuales ${ }^{10}$. Ahora bien, si en verdad no podemos negar la incidencia de estos cambios, especialmente en las regiones y pueblos que rodeaban a Egipto, tampoco podemos olvidar que muchas áreas del país permanecían sin explotar y que, regiones como el Fayum o el Delta, tanto oriental como occidental, no fueron explotadas hasta época Tolemaica. Por otra parte, si bien el descenso en las crecidas del Nilo puede cuantificarse en un $40 \%$, también es cierto que el Nilo siguió constituyendo el único lugar donde podía desarrollarse una agricultura $y$, por tanto, una cultura.

Por otra parte, no podemos dejar sin señalar otra idea asociada al mundo egipcio; su consideración como una unidad geográfica. Es cierto que la dualidad Alto y Bajo Egipto ya entraña una diferenciación evidente, pero no lo es menos que ningún río discurre a lo largo de más de 1.000 $\mathrm{Kms}$. de forma uniforme. Así, las provincias situadas más al Sur, aquellas más cercanas a Nubia, son en las que el río discurre más encajonado, con escasas posibilidades de incrementar la cantidad de tierra susceptible

- Que en el campo antropológico encuentra su reflejo en las tesis de Rappaport (1977) sobre la mala adaptación de las sociedades a unos cambios que se producen en su medio geográfico.

- Sin embargo, el modelo de Service, utilizado para explicar el nacimiento del Estado y los pasos previos a su constitución -banda, tribu y sociedad de jefatura, correspondiendo cada uno a un grado de evolución, adaptación y especialización-, no son válidos para explicar el colapso; siguiendo el esquema evolucionista, si algo nace se desarrolla y después desaparece, lo que surge debe tener un carácter totalmente nuevo, diferente de lo anterior, lo que en el caso de la civilización egipcia no se produce.

10 Una más de las ideas preconcebidas respecto al mundo faraónico es que sus condiciones climatológicas siempre fueron las mismas, asemenjándose en líneas generales a las actuales, lo cual no es cierto. 
de ser cultivada, siendo de las mismas, precisamente, de donde proceden los textos egipcios relativos a la existencia de hambrunas (Kanawati, 1980; Vandier, 1936), y no de todo el país ${ }^{11}$. Esa diversidad regional ha llevado a plantear que los centros que adquieren importancia durante este período son los mismos que habían caracterizado la vida cultural y política de Egipto con anterioridad a la unificación, es decir, lo que se produciría sería una vuelta a la situación previa que había sido superada mediante una gran centralización, no siendo las pirámides más que elementos ideológicos y aglutinadores de una sociedad (Wenke, 1989).

Otro de los aspectos a considerar en relación a la incidencia del medio geográfico es el demográfico, especialmente en dos aspectos; A) cuánta población había en Egipto y, B) cuánta población que estaba en contacto con Egipto se vio afectada en su modo de subsistencia por estos cambios naturales.

A) Cuantificar la población egipcia es difícil (Butzer, 1976), habiéndose realizado recientes estudios sobre el período romano (Bagnall, 1994). La mayoría de los estudios coinciden en señalar en un millón de personas la población total de Egipto (Mortensen, 1991), cifra a todas luces insuficiente para poder hablar, como se ha hecho, de problemas de tierra, aunque sí de rentabilidad, en Egipto.

B) La llamada circunscripción social y económica de Egipto (Carneiro, 1970) ha existido siempre, por lo que el número de poblaciones que vivian en los desiertos que rodean a Kemet es muy escaso. Lógicamente, estas poblaciones también se verían afectadas por los cambios climáticos pero, también es cierto que su economía, nómada y ganadera, se vería menos afectada por un descenso de las crecidas del Nilo, por lo que no todos ellos se verían obligados a emigrar hacia la llanura aluvial, planteamiento que es válido tanto para el desierto del Sinaí como para las poblaciones del desierto oriental.

Respecto al mundo de Nubia, sería la Baja Nubia la región más afectada por el mencionado encajonamiento del Nilo, pero no hemos de olvidar que desde la desaparición del grupo A a comienzos de la I dinastía esta región estaba prácticamente deshabitada y, si bien es cierto que grupos de nubios

11 En relación con lo expresado anteriormente con los textos, muchas veces se olvida dónde es emitido, lo que puede reflejarnos coyunturas regionales determinadas, procediendo a extrapolar lo expresado a toda la cultura. Un ejemplo puede ser la famosa Estela del hambre, atribuída a Djoser pero que fue erigida por Tolomeo II, o los textos biográficos de los nomarcas de los nomos más meridionales, cuyas preocupaciones por el abastecimiento de grano y alimento a sus ciudades en nada se corresponden con las preocupaciones de sus colegas gobernantes más al Norte. 
emigran hacia Egipto, también lo es que son integrados como mercenarios, siendo otra cosa radicalmente diferente los cambios que experimentaron las relaciones de Egipto con una región de la que obtenía productos exóticos y que, progresivamente, va poblándose y estructurándose en torno al grupo C.

Una teoría interesante en lo referido al colapso de los Estados es la de Butzer (1980), en el sentido de que las demandas que genera una clase social o un sector de la población que hasta entonces no había desempeñado función alguna, o si lo había hecho era en unas circunstancias diferentes, provoca un desequilibrio que obliga a modificar las estructuras, pudiéndose llegar al colapso, planteamiento en cierta medida similar al clásico de Eisenstadt (1963), en el sentido de que uno de los problemas al que debe enfrentarse todo Estado es el de las relaciones centro-periferia, entre la corte y las provincias, debiendo existir siempre un equilibrio entre los recursos que emanan de uno y van al otro. Como puede deducirse, éste ha sido el planteamiento dominante para explicar la crisis del Reino Antiguo; una progresiva descentralización que culminó en una pérdida del poder real y una independencia de los gobernadores provinciales ${ }^{12}$. Pero ¿por qué se produce la descentralización?

En el caso de Egipto la interpretación dominante durante décadas ha sido la de los crecientes cultos funerarios de los reyes, que iban confiriendo autonomía y recursos a templos y funcionarios en las provincias. Sin embargo, las mismas habían permanecido infravaloradas desde la III dinastía, posiblemente por el deseo de los reyes de acabar con las luchas internas que habían acontecido hasta entonces, pero a lo largo de más de 200 años de centralización, las mismas irían adquiriendo una progresiva importancia al ser imprescindibles, entre otras cosas, para el mantenimiento de la corte menfita, por lo que dicha descentralización debe ponerse en relación no solo con el deseo de los administradores provinciales de adquirir una autonomía, sino también por la propia dinámica del Estado. Pero más allá de las razones que llevaron a una progresiva descentralización, también hemos de preguntarnos por qué la misma debe ser identificada, muchas veces de forma automática, con un declive o freno a lo vivido anteriormente, como si esas nuevas necesidades, preocupaciones e incluso tensiones, no pudieran fraguar en algo enriquecedor.

12 Como en tantos otros aspectos, la investigación histórica ha recurrido a recoger en algunas ocasiones planteamientos realizados desde cualquiera de las llamadas ciencías auxiliares y aplicarlos a determinados momentos históricos, olvidándose posteriormente de la propia renovación que van sufriendo dichos planteamientos, como en el caso de Eisenstadt (Yoffee \& Cowgill 一eds-, 1988). 
Kaufman (1988), plantea el colapso de los Estados desde la perspectiva de la organización, de la quiebra de una administración central que ya no regula, planifica o vigila la ejecución de una serie de actividades imprescindibles para el funcionamiento del Estado y de las provincias, lo que en el caso del mundo egipcio se ha puesto en relación con la quiebra del sistema que mantenía en funcionamiento la organización hidráulica. También en relación con este planteamiento está el hecho de la progresiva autonomía de los funcionarios locales que, aprovechando la distancia, los problemas de comunicación y sus innatas ansias de autonomia, fueron minando el funcionamiento de un Estado central y burocrático. En el caso de Egipto esta autonomía es cierta, detectamos una progresiva independencia, pero también hemos de recordar que la vida en la corte era muy poco activa, que durante gran parte del Reino Antiguo toda la actividad se centró en Menfis y que la fragmentación que acontece se asemeja mucho a la existente durante el período predinástico.

Respecto al manido control hidráulico, es significativo que el título abridor de canales, o el de inspector de canales no aparezca hasta el Reino Medio (Atzler, 1995) ${ }^{13}$, y aunque el control del medio geográfico ha sido importante desde la cultura Badariense, éste se plasma más en el mundo simbólico, religioso, que en el administrativo ${ }^{14}$.

Otro de los factores que pueden aducirse como causantes de una crisis es la quiebra del comercio, aunque en el caso del Egipto del Reino Antiguo la incidencia en los acontecimientos de este factor sería mínima ya que la actividad comercial fue muy escasa, pese al valor que a veces se le ha querido conferir a expediciones como las de Snefru a Biblos, ciudad que actuó como intermediaria ante Ebla (Matthie, 1978). En lo que se refiere al país de Punt, las menciones al mismo son muy escasas hasta la $\checkmark$ dinastía cuando, significativamente, comienzan a aparecer los llamados "líderes de expedición», como Harduf, cuyo relato nos ilustra de cómo se

13 Es cierto que en objetos protodinásticos como la cabeza de maza de Escorpión se está procediendo a la apertura de un canal, pero ello no constituye una prueba de la importancia del régimen hidráulico, máxime cuando es el único documento real asociado con dicha labor conocido en más de 400 años de historia, y sí del carácter ideológico de estos objetos, presentados al templo como reflejo de un mantenimiento del orden.

14 Un ejemplo de cómo ideas y planteamientos asociados al medio geográfico han ido estableciéndose como dogmas es el del transporte de piedra en barcos, labor que en los manuales puede encontrarse como realizada durante la época de la crecida del Nilo para aprovechar la imposibilidad de trabajar en los campos. Pero se olvida que estos meses son los más difíciles para navegar por el río y cuando se analizan las inscripciones de minas y canteras, observamos que era en esa época cuando se trabajaba en las minas, pero que el transporte se realizaba cuando el nivel del río era más bajo (Eyre, 1987). 
había modificado las relaciones de Egipto con Nubia como consecuencia de la aparición del Grupo C.

Respecto al papel que pueblos o grupos bárbaros pudieron ejercer en los acontecimientos, primero debemos referirnos, brevemente, a la situación en Siria-Palestina ${ }^{15}$. Por un lado, estamos ante una región que lejos de caracterizarse por poblaciones nómadas o violentas, desarrolla desde finales del IV milenio un urbanismo, bien por influencia de Egipto (Bramdl, 1992) o de las colonias Uruk del Norte de Siria (Esse, 1991). Es decir, los movimientos que pueden tener lugar en esta región son los de poblaciones marginales que perturban las rutas comerciales o huyen de situaciones extremas de vida. En ambos casos su número no es importante. En segundo lugar, cuando las estructuras urbanas y palaciales de Siria o Palestina desaparecen, las poblaciones que emigran en modo alguno pueden equipararse con grupos nómadas o bárbaros, sino como portadores de una cultura, de unos hábitos que en modo alguno pueden considerarse como tales. Es cierto que en la VI dinastía comienzan a aparecer las biografías militares, como la de Weni, o escenas en las tumbas representando el ataque a fortalezas que, en algunos casos, llegan a ser defendidas por mujeres, pero en modo alguno pueden entenderse estas escenas como reflejo de una hostilidad permanente, máxime cuando las relaciones y productos egipcios en la región son más abundantes en estos momentos, por lo que estas escenas y textos pueden entenderse como reflejo de conflictos con poblaciones marginales o con pequeños estados y reinos de Palestina meridional que, ante la creciente presencia de Egipto defienden sus intereses, no debiendo olvidar que es en estos momentos cuando aparece el término $a m u$, que ya permanecerá como genérico para referirse a los asiáticos (Redford, 1986b), y que al igual que cualquier término genérico debe entenderse como una referencia general y no concreta ${ }^{16}$.

Es por ello también interesante tener siempre presente qué entendemos por bárbaros o extranjeros, su carácter, número, etc., sin olvidar en modo alguno lo que pueden aportar, ya que como hemos visto esos asiáticos que emigran lo hacen desde centros urbanos que, por otra parte, tienen un mayor desarrollo tecnológico en algunas facetas, como la metalurgia. Es muy fácil explicar las relaciones de un Estado con sus vecinos

15 Un analisis de las relaciones internacionales durante este período ya ha sido realizado en otro trabajo anterior (Pérez Largacha, 1997).

16 En todas las civilizaciones y culturas ha de valorarse también el lenguaje de los textos, de las sociedades que emiten sus ideas y términos como «españoles» o "europeos" responden a generalizaciones que, nosotros sabemos no se corresponden con la realidad, pero que sin embargo están presentes. 
como de guerra y amenaza constante, pero muchas veces se olvida el que la guerra puede ser más cara para los Estados que para los bárbaros, al tener que sostener ejércitos que los bárbaros no tienen, no existiendo además la posibilidad del botín. Por otra parte, esos nómadas realizan en muchas ocasiones actividades que la sociedad no quiere realizar, como la propia guerra ${ }^{17}$ o actividades ganaderas, y sin embargo siguen siendo considerados bárbaros por el mero hecho de ser extranjeros. Respecto a su papel como poblaciones marginales que aprovechan los momentos de crisis para infiltrarse, hay que tener presente que ellos siempre están presentes, por lo que los factores que propiciaron su entrada serían, entre otros, el agotamiento del Estado, pudiéndose adoptar la idea de que los Estados son los causantes del colapso de los Estados y que es en esas circunstancias cuando lo externo penetra, variando su forma de penetrar.

Otro de los factores aducidos para definir períodos históricos es la existencia de buenos o malos gobernantes. En el caso de Egipto es muy difícil adentrarse en esta vía al carecer de fuentes, pero sí al menos resulta significativo que reyes como Keops, cuyo reinado se identifica con la centralización y esplendor de Egipto, pasaran inmediatamente a la memoria como nefastos y crueles, tradición que llegará hasta el mundo clásico con Herodoto.

Otra de las razones aducidas, en el caso del mundo romano, es el cambio religioso, en concreto la adopción y expansión del cristianismo. En el caso de Egipto no es que se produzca un cambio radical, pero sí cualitativo. Así, la llamada democratización funeraria no comienza en el Reino Medio sino en la $V$ dinastía, como las propias menciones a Osiris en los Textos de las Pirámides demuestran ${ }^{18}$. Un aspecto que resulta muy interesante es el paso de una concepción solar a otra osiriaca, máxime cuando Osiris encarna la resurrección, la vegetación y la prosperidad de los campos, por lo que la progresiva importancia del culto a Osiris, tanto en el ámbito funerario como cotidiano, puede estar revelando unas necesidades, un cambio en las creencias provocado por las necesidades que iban apareciendo en el seno de la sociedad egipcia, máxime cuando un cambio climático de esta magnitud no se produce rápidamente.

\footnotetext{
17 Es curioso que una civilización como la egipcia, calificada cai siempre como militarista, especialmente durante el Reino Nuevo, apenas presente textos, históricos o no, valorando la vida militar, al contrario.

18 La propia aparición de los Textos de las Pirámides implican que los reyes ya necesitan de una ayuda para acceder al más allá, no como antes que como dioses en la tierra tenían garantizado su vida eterna.
} 
Por todo lo expresado, observamos que en el caso del Reino Antiguo confluyen una serie de factores que obligan al Estado centralizado a modificar su actuación, a prestar una mayor atención a las provincias, al mundo exterior que le rodea, a la situación interna y a desarrollar una administración provincial que, en un primer momento es claramente dependiente de la corte, pero que con el paso del tiempo irá desvinculándose, no ya por solo por las ansias expansionistas, sino porque los funcionarios locales van asumiendo, y realizando, las tareas que debian ser realizadas por el rey; mantener la prosperidad económica y la seguridad interna, entendiéndose ésta no solo como una lucha contra nómadas o extranjeros que afluyen a Egipto, sino también contra un entorno hostil que amenaza continuamente a la rica llanura aluvial, lucha que es mucho más importante en los nomos meridionales, aquellos en los que las biografías militares y de nobles son más frecuentes y en las que el mantenimiento de Maat es obra de los mismos, anticipando uno de los principales cambios que se producirán en Egipto a partir del Primer Período Intermedio; el mantenimiento de Maat ya no será responsabilidad solo del rey, sino de toda la comunidad, lo que aumentará la conciencia de pertenecer a una misma unidad, Egipto, aun con diferencias regionales.

Llegados a este punto, debemos detenernos, brevemente, en los factores, internos y externos, que provocaron no la crisis, sino el cambio del Reino Antiguo.

\section{A) FACTORES INTERNOS}

El establecimiento de numerosas fundaciones piadosas por parte de los Faraones para el mantenimiento de su culto funerario junto a los privilegios otorgados por los llamados Decretos de Exención, minó el control del Estado sobre importantes partes de Egipto y sus recursos, al mismo tiempo que la administración provincial debilitó la autoridad real en los nomos, actuando los gobernadores como señores de sus territorios.

Estas han sido, en líneas generales, las causas aducidas para el final del Reino Antiguo, aduciéndose como prueba el descenso en el tamaño y calidad de las pirámides, consecuencia de una crisis económica, y de una paulatina perdida de la monarquía del control que hasta entonces ejercía sobre todos y cada uno de los recursos de Egipto. Sin embargo, este modo de "cuantificar" los efectos de una crisis no son validos ya que ese pretendido descenso económico no se corresponde con el hecho de que aun disminuyendo en tamaño y calidad, los complejos pi- 
ramidales presentan una mayor decoración (Arnold, 1997; Roth, 1993). Igualmente, en muchas ocasiones las fundaciones piadosas fueron creadas en zonas que estaban sin explotar, desde el Fayum como en el Delta en tiempos de Snefru, donde se realizó una política de colonización (Moens \& Wetterstram, 1988). La idea de que a medida que las pirámides descendían en tamaño las tumbas de los gobernadores y nomarcas iban siendo más ricas y grandes no es del todo válida, como ya puso de manifiesto Kanawati (1980) y recientes excavaciones en el Alto Egipto han confirmado.

Por otra parte, hemos de pensar en por qué se produce esa descentralización. Por un lado, no podemos olvidar el descenso en el nivel de las crecidas del Nilo, señalando Hassan (1993), que un descenso del 40$50 \%$ en el nivel de las crecidas limita a la mitad la cantidad de grano y si un hombre podía consumir 200 kilos de cereales al año, durante el Primer Período Intermedio solamente podía consumir alrededor de 100, sin poder acudir además al exterior en busca de alimento o a los almacenes estatales. Si en líneas generales el planteamiento de Hassan puede parecer válido, tampoco podemos olvidar que amplias regiones de Egipto permanecían sin explotar, no debiendo olvidar que la importancia que comienza a adquirir Osiris, y especialmente la región de Abidos, se debe a que es la más fértil de todo Egipto, región que, significativamente había experimentado un declive desde el período de unificación como consecuencia de la centralización pero que ahora se recupera. Por otra parte, la incidencia del cambio debe también matizarse ya que los útiles agrícolas siguieron siendo de madera y no se realizó avance técnico alguno que facilitara la irrigación permanente de los campos.

Es cierto que algunas escenas, como las del corredor de la pirámide de Unas, nos presentan a hombres famélicos que podrían ponerse en relación con este descenso, pero también lo es que se puede estar representando a gentes procedentes del desierto, o bien ser escenas propagandísticas representando lo que el buen gobierno de estos reyes evita, ya que es a partir de este momento cuando comienzan a ponerse las bases de la propaganda real.

Mención aparte merecen las conspiraciones y luchas internas por el poder. La descentralización administrativa de Egipto a partir de la $\mathrm{V}$ dinastía es una realidad, del mismo modo que la existencia de rivalidades internas entre la familia real (final de la IV dinastía), por lo que el deterioro de la situación económica pudo influir en la aparición de tensiones internas, tanto entre la familia real como entre ésta y los gobernadores provinciales, más preocupados en ocasiones de garantizar el sustento a 
su población que por satisfacer las necesidades de la administración central.

El que la agricultura y la economía fueran deteriorándose debió de influir de forma muy importante en la visión de la realeza y en la valoración de los gobernadores provinciales, debiendo hacer estos últimos frente a la realidad cotidiana. Al respecto mucho se ha escrito sobre personajes como Ankhifty o Heqaib, nomarcas que posteriormente gozaron de una gran reputación en Egipto y cuyo culto y memoria fue mantenido durante siglos. Pero junto a estos personajes cuyas acciones contribuyeron a solucionar el hambre y la seguridad física de sus territorios, debieron de existir muchos otros gobernadores, cuya memoria y acciones no fueron recordadas.

\section{B) FACTORES EXTERNOS}

Un cambio de esta magnitud no incide en una única región. En Mesopotamia y Siria-Palestina también se observa una desecación del clima que, en el caso de Mesopotamia, acelera el proceso por el que el centro político, administrativo y comercial va trasladándose al norte de Siria, modificaciones que conllevan la aparición de movimientos migratorios que, en el caso de Egipto, pudieron influir en la política exterior a partir de la $V$ dinastía $y$, especialmente, en el cambio que se detecta en las relaciones entre Nubia y Egipto. Tampoco podemos olvidar a todas aquellas poblaciones que, establecidas con anterioridad en regiones donde podían practicar una agricultura de subsistencia o una actividad ganadera, se verían afectadas por este cambio afluyendo a Egipto como única salida a su pobreza.

Estas poblaciones emigran con todas sus pertenencias y ofrecen a la administración central o local aquello que tienen, sus brazos para ser utilizados como mercenarios o sus conocimientos técnicos en el caso de poblaciones que emigran de centros urbanos.

Es por todo ello que el colapso del Reino Antiguo, como el de otros grandes Estados o imperios, ha sido interpretado en los últimos años como una consecuencia de las necesidades de reorganización administrativa, política y económica que tuvo que realizar Egipto a partir de la $\mathrm{V}$ dinastía. La aparición de nuevas necesidades, como garantizar la seguridad de unas fronteras a las que podían afluir poblaciones que huían de su pobreza propicio que un pequeño contingente militar se desarrollara, con la incidencia que el mismo puede tener como poder fáctico en determina- 
dos momentos, no debiendo olvidar que según la tradición Teti fue asesinado por su guardia personal.

La necesidad de acceder mediante intermediarios a los productos exóticos, tanto en Siria-Palestina como en Nubia, quebró otra de las bases sobre las que se basa el poder de un Estado, su control de las materias primas que requiere para sus ritos, ceremonias o los mismos útiles de trabajo.

A todo esto hay que unir la descentralización administrativa, con unos funcionarios que debían hacer frente a nuevas necesidades y coyunturas en un Estado en el que las comunicaciones, aun a pesar del Nilo, no serían lo suficientemente fluidas, sin olvidar los deseos de toda gobernante de ir adquiriendo nuevas cuotas de poder. Al respecto resulta significativo que durante la XII dinastía, la política de los Faraones respecto a la administración provincial vaya encaminada en dos direcciones, primero a recuperar la potestad del Faraón de realizar él las levas militares y, en segundo lugar, evitar la heredabilidad de los cargos y la consiguiente consideración de los mismos como algo propio.

Es decir, la crisis del Reino Antiguo no se debió a un único factor, sino a la confluencia de una serie de circunstancias que, es cierto, dieron paso a una importante inestabilidad política, pero también lo es que la misma no se produjo hasta finales de la VI dinastía y que este período de desorden duro relativamente poco, demostrándonos igualmente el dinamismo de la propia civilización egipcia. La ideología desarrollada por los primeros Faraones era valida, y así fue establecida, para un marco cultural, social y económico determinado, siendo lógico que cuando se producen cambios en dicho marco comiencen a aparecer fisuras.

Un último aspecto a mencionar, y no suficientemente valorado en la investigación hasta el momento, es la coincidencia, si se puede hablar de ella en la reconstrucción histórica, de las crisis que se producen en Egipto, Siria-Palestina y Mesopotamia. No es que los planteamientos de Wallerstein de un sistema mundial puedan y deban extrapolarse a la esfera política, además de la económica, pero si es cierto que el camino iniciado hace pocos años en la egiptología de valorar y considerar las fuentes externas esta abriendo un cuadro histórico más rico que, además, comienza a encontrar su reflejo material no solo en los textos y su nueva valoración, también en el registro arqueológico del Bajo Egipto, región que siempre fue, hasta época romana y fatimi, el verdadero motor de un país que como Egipto depende del Nilo y sus crecidas para el desarrollo de la agricultura, pero que es mucho más que una mera administración preocupada por construir canales, tumbas y templos. 


\section{BIBLIOGRAFIA}

ARNOLD, D. (1997): "Royal cult complexes of the Old and Middle Kingdoms", Temples of Ancient Egypt, B. Schafer (Ed.), Chicago, 31-84.

AssmanN, J. (1995): Egipto a la luz de una teoría pluralista de la cultura, Madid.

ATZLER, M. (1995): "Some remarks on interrelating environmental changes and ecological, socio-economic problems in the development of the Early Egyptian inundation culture", Archaeo-Nil 5, 7-65.

Bagnall, R. (1994): The Demography of Roman Egypt, Cambridge.

BAINES, J. (1987): "Practical Religion and Piety", JEA 73, 79-98.

BARTA, W. (1975): Untersuchungen zur Göttlichkeit des regierenden Königs: Ritus und Sakrat- * königtum nach Zeugnissen der Frühzeit und des Alten Reiches, Berlín.

BELL, B. (1971): "The Dark ages in ancient history. The first Dark Age in Egypt», AJA 75, 1-26.

BIETAK, M. (1996): Avaris. The capital of the Hyksos, Londres.

BourRIAU, J. (1991): "Patterns of Change in burial customs during the Middle Kingdom», Middle Kingdom Studies, S. Quirke (Ed.), Londres 3-20.

BrandL, B. (1992): «Evidence for Egyptian colonization of the Southern Coastal plain and Lowlands of Canaan during the Early Bronze I pLabor in the Ancient Near Easte, Chicago 447.riod", The Nile Delta in transition: 4th-3rd. Millennium B.C., Tel Aviv, 441-76.

BUTZER, K. (1976): Early Hidraulic civilization in Egypt, Chicago.

- (1980): "Civilizations: organisms or Systems?", American Scientist 68, 517-23.

CARNeIRO, R. (1970): «A Theory of the origins of the State», Science 169, 733-38.

EISENSTADT, S. (1969): The Political Systems of Empires: the Rise and Fall of the Historical Bureaucratic Societies, Nueva York.

ESSE, D. (1991): Subsistence, trade, and social change in Early Bronze Age Palestine, Chicago.

EYRE, C. (1987): "Work and the organization of work in the Old Kingdom".

GABALLA, G. (1976): Narrative in Egyptian Art, Berlín.

Griffiths, G. (1991): The Divine Verdict. A study of divine Judgement in the Ancient Religions, Leiden.

HASSAN, F. (1993): «Town and Vilage in Ancient Egypt: ecology, society and urbanization», The Archaeology of Africa, Londres, 551-69.

- (1997): «The dynamics of a riverine civilization: a geoarchaeological perspective on the Nile Valley, Egypt», World Archaeology 29, 51-74.

HoffmeIER, J. (1991): «The coffins of the Middle Kingdom: the residence and the regions», Middle Kingdom Studies, S. Quirke (Ed.), Londres, 69-86.

KANAWATI, N. (1980): Gubernamental reforms in the Old Kingdom, Londres.

KAUFMAN, H. (1988): «The collapse of Ancient States and civilizations as an organizational problem", The Collapse of Ancient States and Civilizations, N. Yoffe \& G. Cowgill (Eds.), Chicago, 219-35.

LEHNER, M. (1997): The Complete Pyramids, Londres.

MATTHIAE, G. (1978): «Inscriptions royales egyptiennes de l'ancien empire a Ebla", Mesopotamien und seime nachbasr, 125-31.

MOESS, M. \& WETTERSTROM, W. (1988): «The Agricultural Economy of and Old Kingdom town in Egypt's west Delta», JNES 47, 159-74.

MORTENSEN, B. (1991): "Change in the Settlement pattern and population in the Beginning of the Historical period", Ägypten und Levante 2, 11-37.

O'ConNoR, D. \& Silverman, D. (Eds) (1995): Ancient Egyptian Kingship, Leiden.

Pérez LaRgacha (1998a): «Egipto y el Próximo Oriente. Perspectivas de trabajo y colaboración”, Isimu 1, 247-54.

- (1998b): Egipto en tiempos de las Pirámides, Madrid.

- (en prensa): «Egypt and the Ancient Near East», JNES.

Posener, G. (1960): De la divinité du Pharaon, París.

RAPPAPORT, R. (1977): «Maladaptation in Social Systems», The Evolution of Social Systems, J. Friedman \& M. Rowlands (Eds.), Londres 49, 73.

Redford, D. (1986): Pharaonic King-list, Annals and Day-books; a contribution to the Study of the Egyptian Sense of History, Toronto.

- (1986b): «Egypt and Western Asia in the Old Kingdom», JARCE 23, 125-43. 
Roth, A. (1993): «Social Change in the Fourth Dynasty; the spatial organization of pyramids, tombs and cemeteries", JARCE 30, 33-55.

SERVICE, E. (1981): Los orígenes del Estado y la Civilización, Madrid.

VANDIER, J. (1936): Le famine dans l'Egypte ancienne, El Cairo.

WENKE, R. (1989): "Egypt origins of complex societies", Annual Review of Anthropology 18, 129-55.

YofFE, N. \& Cowgill, G. (Eds.) (1988): The colapse of Ancient States and Civilizations, Chicago. 